\title{
THE EFFECT OF SINTERING CONDITIONS ON THE MICROSTRUCTURE AND ELECTRICAL PROPERTIES OF Pb( $\left.\mathrm{Zr}_{0.52} \mathrm{Ti}_{0.48}\right) \mathrm{O}_{3}$ CERAMIC
}

\section{Nur ShafizaAfzan Sharif ${ }^{1}$, Julie Juliewatty Mohamed ${ }^{1}$, Hutagalung Sabar Derita ${ }^{1}$, Zainal Arifin Ahmad ${ }^{1}$, Mohd. Zulkifly Abdullah ${ }^{2}$, Hasmaliza Mohamad ${ }^{1}$ and Wan Azhar Wan Yusoff ${ }^{3}$}

\author{
${ }^{1}$ School of Materials and Mineral Resources Engineering, \\ USM Engineering Campus, 14300 NibongTebal, Penang, Malaysia \\ Email: shafiza_sharif@yahoo.com ${ }^{\mathrm{a}}$, srjuliewatty@eng.usm.my ${ }^{\mathrm{b}}$, \\ ${ }^{2}$ School of Mechanic Engineering, USM Engineering Campus, \\ 14300 NibongTebal, Penang, Malaysia \\ Email:mezul@eng.usm.my ${ }^{\mathrm{e}}$ \\ ${ }^{3}$ Faculty of Manufacturing Engineering, \\ UMP Kampus Pekan, 26600 Pekan, Pahang, Malaysia \\ Email: wawy.engineer@gmail.com ${ }^{\mathrm{g}}$
}

\begin{abstract}
Lead zirconatetitanate $\mathrm{Pb}\left(\mathrm{Zr}_{0.52} \mathrm{Ti}_{0.48}\right) \mathrm{O}_{3}$, (PZT) from a mixture of commercial $\mathrm{PbO}$, $\mathrm{TiO}_{2}$ and $\mathrm{ZrO}_{2}$ powders was successfully prepared using a planetary ball mill. The microstructure and electrical properties of PZT ceramic were found to be highly sensitive to the sintering condition. The influence of microstructure and electrical properties on the sintering condition of the samples was studied. SEM analysis indicated that a shorter sintering time with higher sintering temperature promotes fine structure and densification. This was proves where the relative density of the sintered PZT ceramics obtained was measured to be approximately $99 \%$ of the theoretical density. The electrical properties of the ceramic sintered at a higher temperature with a different sintering time were measured at high frequencies. The result revealed that different sintering conditions have a big impact on electrical behavior in a broad frequency region.
\end{abstract}

Keywords: PZT; sintering; microstructure; electrical.

\section{INTRODUCTION}

Lead zirconatetitanate (PZT) is the most researched material in the perovskite oxide ferroelectric family, and also the most technologically important material in the electroceramic industry (James et al., 2008). The application of piezoelectric materials is almost unlimited and depends only on the skill of the users. It is sufficient to mention the newest and most exciting applications of such ceramics, in piezoelectric micromotors, microrobots, actuators, and the control of displacement and positioning systems, to understand the importance of piezoelectric materials with improved characteristics (Tănăsoiu, Dimitriu, \& Miclea, 1999). Researchers also claim that relax or ferroelectrics with $\mathrm{AB}_{(1-\mathrm{x})} \mathrm{B}_{\mathrm{x}} \mathrm{O}_{3}$ type perovskite has attracted much attention because of their excellent dielectric and electromechanical properties. $\mathrm{Pb}\left(\mathrm{Zr}_{0.52} \mathrm{Ti}_{0.48}\right) \mathrm{O}_{3}$ belongs to the perovskite structural family with the general formula $\mathrm{ABO}_{3}(\mathrm{~A}=$ mono or divalent ions, $\mathrm{B}=$ tri- to pentavalent cations). It is well established that the physical properties or device parameters of PZT can be tailored by synthesizing the materials with improved 
processing techniques and making suitable substitutions of A and/or B sites. The electrical properties of relax or ferroelectrics are greatly influenced by the manner in which the B site cations (Band B ions) are distributed and ordered on the B site sublattice. The $\mathrm{Zr} / \mathrm{Ti}$ ratio is known to strongly influence properties such as the elastic constant and the dielectric constant (Eitssayeam, Intatha, Rujijanagul, Pengpat, \& Tunkasiri, 2006).

The present investigation was therefore devoted to studying the effect of sintering conditions on the main properties of $\mathrm{Pb}\left(\mathrm{Zr}_{0.52} \mathrm{Ti}_{0.48}\right) \mathrm{O}_{3}$ materials. Cheng et al. (2009) investigated whether the dielectric constant of ceramic materials were highly dependent on the processing conditions such as milling time, sintering temperature and duration of the sintering. The dielectric constant was reported to be 2000 to 80000 by varying the sintering condition, and ascribed to giant barrier layer effects at grain boundary (Fang \& Chung, 2011; Ramesh, Tan, Amiriyan, \& Teng, 2011; Sivakumar, Ramesh, Chin, Tan, \& Teng, 2011). Kim et al. (2001) studied whether microstructural features such as grain size and grain boundary layer thickness of ceramic materials had strong dependence on the sintering duration at elevated temperatures. This paper will describe details of the influence of sintering conditions on the microstructural and electrical characteristics of $\mathrm{Pb}\left(\mathrm{Zr}_{0.52} \mathrm{Ti}_{0.48}\right) \mathrm{O}_{3}$.

\section{EXPERIMENTAL DETAILS}

$\mathrm{Pb}\left(\mathrm{Zr}_{0.52} \mathrm{Ti}_{0.48}\right) \mathrm{O}_{3}$ was synthesized using the high planetary ball mill reaction method. $\mathrm{A}$ high purity (99.9\%) starting powder of $\mathrm{PbO}$ (Aldrich), $\mathrm{ZrO}_{2}$ (Merck) and $\mathrm{TiO}_{2}$ (Merck), powders with $10 \mathrm{wt} \%$ excess $\mathrm{PbO}$ was used. The milling process was carried out in a Retsch planetary ball mill for 60 hours at room temperature. A $250 \mathrm{ml}$ tungsten carbide vial and 50 tungsten carbide balls with a diameter of $10 \mathrm{~mm}$ were used as milling media. The mixture without any additive was placed in the vial with a ball to powder weight ratio of 10:1. The milling speed was set at 200rpm and was stopped for 15 minutes every 30 minutes to cool down the milling system. After milling, the powder was ground and pressed into pellets using hydraulic pressing at a pressure of $110 \mathrm{MPa}$. Finally, the pellets were sintered at $1200^{\circ} \mathrm{C}$ for 1,2 and 3 hours with both heating and cooling rates being fixed at $5^{\circ} \mathrm{C} / \mathrm{min}$. The phase formations were characterized using an XRD Bruker AXS D8 Diffractometer with $\mathrm{Cu} \mathrm{K \alpha}$ radiation in a scan between $10^{\circ}$ to $90^{\circ}$. The microstructure was observed using the Variable Pressure Field Emission SEM (VPFESEM Zeiss Supra ${ }^{\mathrm{tm}}$ 35VP) and density was measured using the Archimedes principle. Before measuring the electrical properties, the pellets were ground with $\mathrm{SiC}$ paper to produce a flat uniform surface and painted on both sides with silver electrode. Meanwhile, the dielectric response of the samples was measured with a precision Impedance Analyzer (model: RF Impedance/Material Analyzer 4291B Hewlett Packard) over a frequency range from $1 \mathrm{MHz}$ to $1 \mathrm{GHz}$ at an oscillation voltage of $500 \mathrm{mV}$.

\section{RESULTS AND DISCUSSION}

The XRD results were analyzed in this study. XRD spectrums for all samples recorded at room temperature are shown in Figure 1. All samples sintered for different durations show a pure single phase and match the standard Inorganic Crystal Structure Database (ICSD) in X'pert High Scores Plus software (ICSD File No. 98-007-6143).It was found that all samples exhibit a tetragonal crystal structure. From the measurement certainty, there is no obvious difference in lattice parameters between the samples. No secondary 
phase was found in the XRD pattern which indicated that the samples sintered at 1200 ${ }^{\circ} \mathrm{C}$ for 1 hour were already reacted into PZT phase during the sintering process. Other than that, the intensity of diffraction peaks for PZT perovskite phase increased as the sintering soaking times increased. This phenomenon indicated that the crystallinity of PZT samples becomes better and improves the particle size (Tripathi et al., 2009).

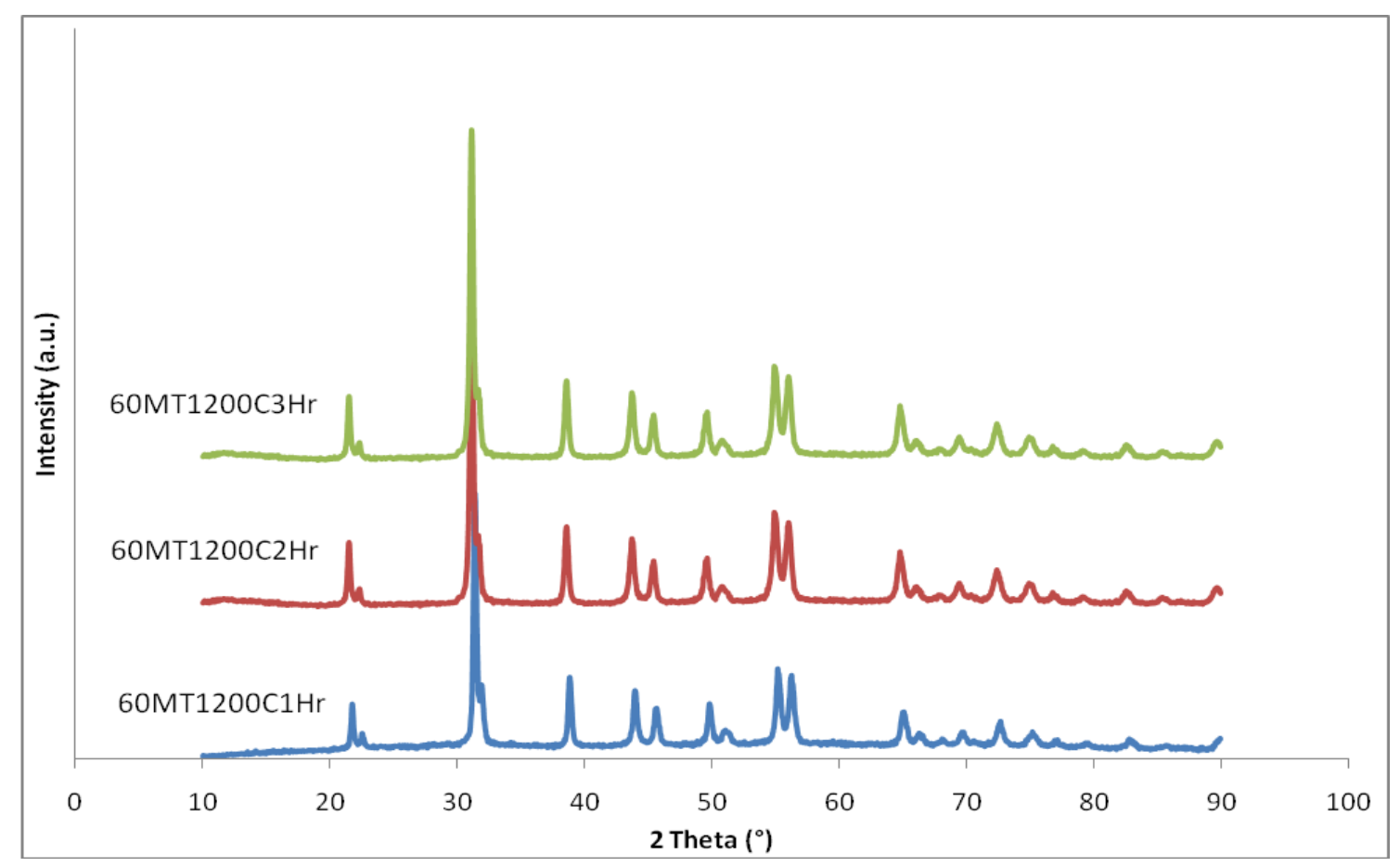

Figure 1. XRD pattern of PZT samples with different sintering durations.

Figure2 shows the SEM micrographs of PZT samples sintered for different sintering durations. A porous microstructure with a small grain size is observed in the sample sintered at $1200^{\circ} \mathrm{C}$ for 1 hour. Increasing the sintering duration significantly promotes grain growth and microstructural densification with a minimal porosity which can be seen in Table 1. Samples sintered fir2 hours show fairly uniform grain sizes with clear grain boundaries. These samples show the densest microstructure with a grain size range of $1-3 \mu \mathrm{m}$. The grain growth in the sample sintered at $1200^{\circ} \mathrm{C}$ for longer soaking times implies an increase in the length of liquid phase and makes the grain larger. At this sintering time (3 hours), the sample density drops to $93 \%$ of the theoretical density. This phenomenon is believed to be attributed to the loss of $\mathrm{PbO}$ as well as the coarsened grain (Kong, Ma, Zhu, \& Tan, 2002).

Table 1. Density measurement of PZT samples.

\begin{tabular}{lcccc}
\hline Sample & Air $(\mathrm{g})$ & Liquid $(\mathrm{g})$ & Density $\left(\mathrm{g} / \mathrm{cm}^{3}\right)$ & Relative Density $(\%)$ \\
\hline 60MT1200C1Hr & 0.948 & 0.826 & 7.768 & 97.08 \\
60MT1200C2Hr & 1.144 & 0.9996 & 7.927 & 99.06 \\
60MT1200C3Hr & 0.958 & 0.8305 & 7.516 & 93.93 \\
\hline
\end{tabular}



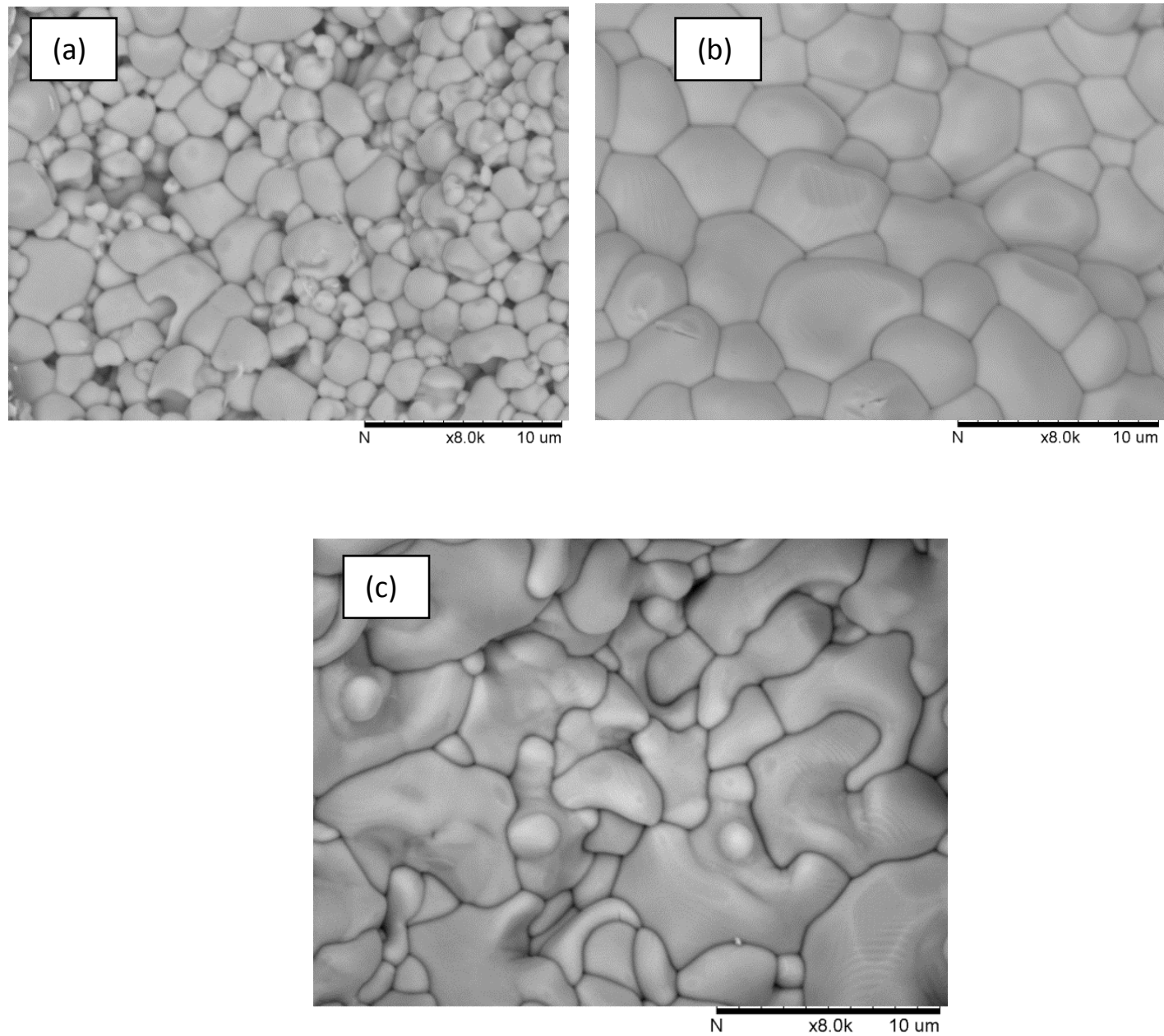

Figures 2. SEM micrograph of PZT samples sintered at $1200{ }^{\circ} \mathrm{C}$ for, (a) $1 \mathrm{~h}$, (b) $2 \mathrm{~h}$, (c) $3 \mathrm{~h}$

Figure 3 shows the results of dielectric constant and dielectric loss at frequency $1 \mathrm{MHz}$ of the samples sintered at $1200^{\circ} \mathrm{C}$ with different soaking times. The dielectric constant and dielectric loss of PZT ceramic materials has been incorporated into both figures for comparison. The figure shows that a sharp change in dielectric constant occurs in the PZT sample sintered for 2 hours. This phenomena occurs due to the related grain structure of the sintered ceramic (Figure 2) and agrees well with the result reported by (Mousharraf \& Islam, 2013; Randall, Kim, Kucera, Cao, \& Shrout, 1998) where the maximum dielectric constant of PZT ceramics decreases with the decrease of grain size. In Figure 3, at $1 \mathrm{MHz}$, the dielectric constant values for the samples sintered at $1200^{\circ} \mathrm{C}$ for 1,2 and 3 hours were 173,6068 and 686 , respectively. The dielectric loss is closely related to the mechanism of the dielectric response. Based on the widely accepted internal barrier layer capacitance model, the dielectric loss mainly originates from the conductivity of the electro ceramic conducting crystalline grain/subgrain as well as the insulating barrier. The conductivity of the barriers leads to the leakage loss. As known, ferroelectric material exhibits a high dielectric constant with higher dielectric loss (Adams, Sinclair, \& West, 2002). In order to lower the value for dielectric loss, investigation ofthe sintering soaking times has been undertaken. The dielectric loss decreased when increasing the soaking time. This behavior indicates that 
different sintering times significantly improve the dielectric constant and greatly reduce the value for dielectric loss. The dielectric loss is around 0.008-0.04, and the value starts to increase at higher frequency. The dielectric loss obtained in this study was much lower than in previous results (Kong et al., 2002).

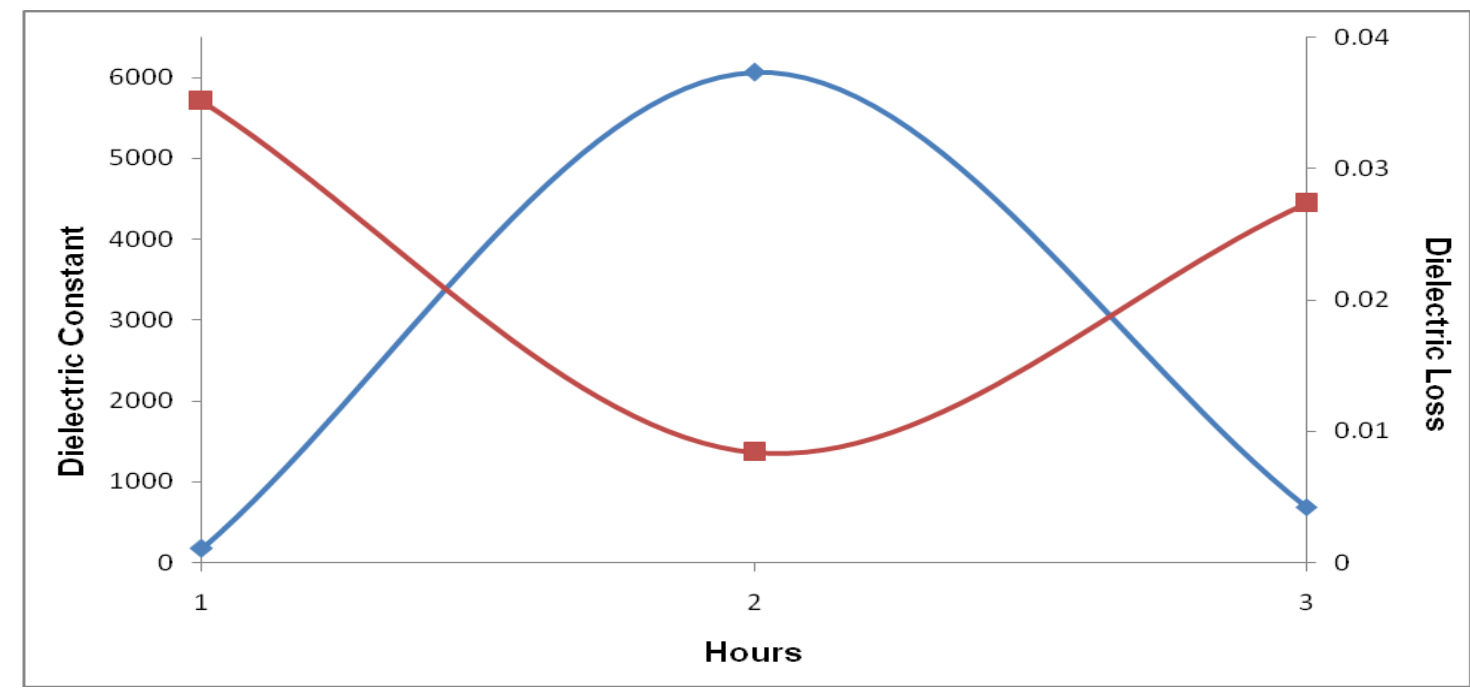

Figures 3. Dielectric properties of the sintered PZT ceramics as a function of sintering times

\section{CONCLUSION}

It is crucial to adequately control sintering times to obtain the desired microstructure and dielectric properties. The sample sintered at $1200^{\circ} \mathrm{C}$ for 2 hours shows a clear grain and densemicrostructure. The sample sintered at $1200^{\circ} \mathrm{C}$ for 3 hours also shows melting grains, which happens due to the loss of $\mathrm{PbO}$. The effects of different sintering conditions on density, phase formation, microstructure and dielectric properties were discussed. Increasing sintering times enhances the density (99\% of the theoretical density), however in longer sintering times (3 hours), the density drops to $93 \%$ of the theoretical density. The highest dielectric constant (6068) with lower dielectric loss $(0.008)$ with clear uniform grain was obtained by the sample sintered at $1200^{\circ} \mathrm{C}$ for 2 hours.

\section{ACKNOWLEDGEMENTS}

The authors would like to thank the Universiti Sains Malaysia for financial support under grant no. 60310015 and the Ministry of Higher Education of Malaysia under grant no 6730071.

\section{REFERENCES}

Adams, T. B., Sinclair, D. C., \& West, A. R. (2002). Giant barrier layer capacitance effects in cacu 3 ti 4 o 12 ceramics. Advanced Materials, 14(18), 1321-1323.

Cheng, B., Lin, Y.-H., Yuan, J., Cai, J., Nan, C.-W., Xiao, X., \& He, J. (2009). Dielectric and nonlinear electrical behaviors of la-doped cacu 3 ti 4 o 12 ceramics. Journal of Applied Physics, 106(3), 034111-034114. 
Eitssayeam, S., Intatha, U., Rujijanagul, G., Pengpat, K., \& Tunkasiri, T. (2006). Structural and electrical properties characterization of $(1-\mathrm{x})$ pbzr0. 52ti0. 48o3xbafe0. 5nb0. 5 o3 system. Applied Physics A, 83(2), 295-299.

Fang, T.-T., \& Chung, H.-Y. (2011). Reassessment of the impedance spectra and dielectric responses of undoped and casio3-doped cacu3ti4o12. Journal of Applied Physics, 109(1), 014102.

James, A., Rao, B. C., Kamat, S., Subrahmanyam, J., Srinivas, K., \& Thakur, O. (2008). Structural, thermal, electromechanical and mechanical property studies on (pb0. 93la0. 07)(zr0. 60ti0. 40) o3 ceramics synthesized by a high energy mechanochemical milling process. Smart Materials and Structures, 17(3), 035020.

Kim, B.-G., Cho, S. M., Kim, T.-Y., \& Jang, H. M. (2001). Giant dielectric permittivity observed in pb-based perovskite ferroelectrics. Physical review letters, 86(15), 3404-3406.

Kong, L., Ma, J., Zhu, W., \& Tan, O. (2002). Phase formation lead zirconate titanate via a high-energy ball milling process. Journal of materials science letters, 21(1), 25-27.

Mousharraf, A., \& Islam, M. F. (2013). Effect of ta2o5 doping on the microstructure and dielectric properties of batio3 based ceramics. International Journal of Automotive and Mechanical Engineering, 7, 840-849.

Ramesh, S., Tan, C. Y., Amiriyan, M., \& Teng, W. D. (2011). Sintering effects on the densification of nanocrystalline hydroxyapatite. International Journal of Automotive and Mechanical Engineering, 3, 249-255.

Randall, C. A., Kim, N., Kucera, J. P., Cao, W., \& Shrout, T. R. (1998). Intrinsic and extrinsic size effects in fine-grained morphotropic-phase-boundary lead zirconate titanate ceramics. Journal of the American Ceramic Society, 81(3), 677-688.

Sivakumar, S., Ramesh, S., Chin, K. L., Tan, C. Y., \& Teng, W. D. (2011). Effect of sintering profiles on the properties and ageing resistance of y-tzp ceramic. International Journal of Automotive and Mechanical Engineering, 4, 405-413.

Tănăsoiu, C., Dimitriu, E., \& Miclea, C. (1999). Effect of nb, li doping on structure and piezoelectric properties of pzt type ceramics. Journal of the European Ceramic Society, 19(6), 1187-1190.

Tripathi, R., Dogra, A., Srivastava, A., Awana, V., Kotnala, R., Bhalla, G., \& Kishan, H. (2009). Influence of sintering temperature and oxygen annealing on transport properties of la0. 67ca0. 33mno3. Journal of Physics D: Applied Physics, 42(2), 025003. 